Publ. Mat. 62 (2018), 185-211

DOI: 10.5565 /PUBLMAT6211810

\title{
A TRACE THEOREM FOR BESOV FUNCTIONS IN SPACES OF HOMOGENEOUS TYPE
}

\author{
Miguel Andrés Marcos
}

\begin{abstract}
The aim of this paper is to prove a trace theorem for Besov functions in the metric setting, generalizing a known result from A. Jonsson and $\mathrm{H}$. Wallin in the Euclidean case. We show that the trace of a Besov space defined in a 'big set' $X$ is another Besov space defined in the 'small set' $F \subset X$. The proof is divided in three parts. First we see that Besov functions in $F$ are restrictions of functions of the same type (but greater regularity) in $X$, that is we prove an extension theorem and mention examples where this theorem holds. Next, as an auxiliary result that can also be interesting on its own, we show that the interpolation between certain potential spaces gives a Besov space. Finally, to obtain that Besov functions in $X$ can in fact be restricted to $F$, a restriction theorem, we first prove that this result holds for functions in the potential space, and then by the interpolation result previously shown, it must hold in the Besov case. For the interpolation and restriction theorems, we make additional assumptions on the spaces $X$ and $F$, and on the order of regularity of the functions involved. We include an interesting example of our trace theorem, not covered by the classical one.
\end{abstract}

2010 Mathematics Subject Classification: Primary: 43A85.

Key words: Besov spaces, spaces of homogeneous type, trace theorem, extension theorem, restriction theorem, interpolation.

\section{Introduction}

In the Euclidean context, functions in Sobolev spaces $H^{k}$ in $\mathbb{R}^{n}$ can be restricted to subspaces of smaller dimension, obtaining again Sobolev functions. For instance

$$
\left.H^{k}\left(\mathbb{R}^{n+1}\right)\right|_{\mathbb{R}^{n}}=H^{k-1 / 2}\left(\mathbb{R}^{n}\right) .
$$

As shown by A. Jonsson and H. Wallin in [13], these results can be generalized considering Besov spaces, proving Besov functions in $\mathbb{R}^{n}$ leave a Besov trace in certain subsets $F$ of dimension $d<n$ referred to

The author was supported by Consejo Nacional de Investigaciones Científicas y Técnicas, Agencia Nacional de Promoción Científica y Tecnológica and Universidad Nacional del Litoral. 
as $d$-sets (the first noted as $\Lambda_{\alpha}^{p, q}\left(\mathbb{R}^{n}\right)$ and the second one $B_{\beta}^{p, q}(F)$ in their work):

$$
\left.B_{p, q}^{\alpha}\left(\mathbb{R}^{n}\right)\right|_{F}=B_{p, q}^{\beta}(F)
$$

with $\beta=\alpha-\frac{n-d}{p}$.

This notation means there exist two bounded linear operators: a restriction operator $\mathcal{R}: B_{p, q}^{\alpha}\left(\mathbb{R}^{n}\right) \rightarrow B_{p, q}^{\beta}(F)$ and an extension operator $\mathcal{E}: B_{p, q}^{\beta}(F) \rightarrow B_{p, q}^{\alpha}\left(\mathbb{R}^{n}\right)$ such that $\mathcal{R} f=\left.f\right|_{F}$ and $\left.\mathcal{E} g\right|_{F}=g$, the precise meaning of these 'pointwise restrictions' described below.

In this paper we attempt to generalize this result from Jonsson and Wallin to the metric setting. We will prove, under certain conditions, that

$$
\left.B_{p, q}^{\alpha}(X)\right|_{F}=B_{p, q}^{\beta}(F)
$$

holds for spaces of homogeneous type, where $F \subset X$ is a closed subset of 'smaller dimension'.

Several recent results deal with extension and restriction operators for Besov and Sobolev spaces in the metric setting. For instance, in [8] the authors give a restriction theorem from the space $X$ to a compact subspace $K$ of lesser dimension for homogeneous Sobolev spaces, obtaining that the restricted functions are in a Besov space. On the other hand, in [11] the authors obtain necessary and sufficient conditions for the existence of an extension operator for Besov spaces, between a domain $\Omega$ and the whole space $X$ ( $\Omega$, being open, has the same dimension as $X)$. In both cases the authors make use of interpolation results between $L^{p}$ spaces and Sobolev spaces, proved in the same papers.

In order to generalize the theorem from Jonsson and Wallin, our work follows a similar path to the one found in [13]. Unlike their case, it was necessary to prove several classical results in our more general context, developing tools that can be relevant in other contexts.

To define the extension operator, the main tool the authors use in [13] consists in partitioning the complement of $F$ in Whitney cubes, with diameter comparable to its distance to $F$, and building with those cubes a smooth partition of unity. To prove that $\mathcal{E} f: X \rightarrow \mathbb{R}$ is actually an extension of $f: F \rightarrow \mathbb{R}$, they show that the restriction

$$
\left.\mathcal{E} f\right|_{F}(t)=\lim _{r \rightarrow 0} f_{B(t, r)} \mathcal{E} f(x) d x
$$

exists and equals $f(t)$ for $\mu$-almost every $t \in F$ (this clearly coincides with the pointwise restriction of $\mathcal{E} f$ when it is continuous). 
As Whitney's Lemma holds in the context of spaces of homogeneous type (see [1]), this result can be generalized, and we obtain in Theorem 2.1 the existence of a linear bounded extension operator

$$
\mathcal{E}: B_{p, q}^{\beta}(F, \mu) \rightarrow B_{p, q}^{\alpha}(X, m),
$$

where $\alpha=\beta+\gamma / p$, for the whole range $0<\alpha<1$ and $1 \leq p<\infty, 1 \leq$ $q \leq \infty$, for the case that $(X, m)$ and $(F, \mu)$ are spaces of homogeneous type with $F \subset X$ closed, $m(F)=0$ and $m$ and $\mu$ satisfy the quotient relation

$$
\frac{m(B)}{\mu(B)} \sim r^{\gamma}
$$

for balls $B$ centered in $F$ with radius $r<2 \operatorname{diam}(F)$. Observe that this relation, although similar, does not imply that either space is Ahlforsregular, see Remark 2.2 .

The strategy described by Jonsson and Wallin to prove the restriction theorem is to use the boundedness properties of the kernel $G_{\alpha}$ of the Bessel potential $\mathcal{J}_{\alpha}$ to guarantee the existence of a bounded operator $\mathcal{R}$ from the Sobolev potential space $\mathcal{L}^{\alpha, p}$ and a Besov-like space $l_{\infty}^{\beta}(A)$, with $A=L^{p}\left(|s-t|^{-d} d \mu(s) d \mu(t)\right)$, such that $\mathcal{R} f=\left.f\right|_{F}$ for continuous functions (which are dense in $\mathcal{L}^{\alpha, p}$ ), and then via an interpolation argument conclude the result

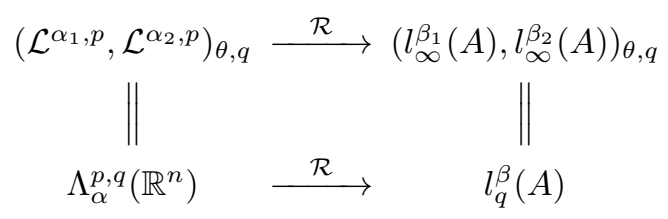

where $l_{q}^{\beta}(A) \cong B_{p, q}^{\beta}(F)$.

To generalize this part, our setting will be Ahlfors regular spaces, $X$ being $N$-dimensional and $F d$-dimensional, $d<N$. Using the potential-Sobolev spaces $L^{\alpha, p}$ constructed in [17], we show that for small orders of regularity, the interpolation between two potential spaces gives a Besov space, generalizing the result from $\mathbb{R}^{n}$ (see the work of Peetre, $[\mathbf{1 9}]$ ), the precise statement found in Theorem 2.4. To prove this we use a continuous version of Calderón's reproducing formula, therefore the approach is different from the result of Peetre.

We then proceed to prove first a restriction theorem for potential functions (Theorem 2.5), and conclude (by the interpolation result) that for small orders of regularity the result holds for Besov functions, meaning there exist a linear bounded restriction operator

$$
\mathcal{R}: B_{p, q}^{\alpha}(X, m) \rightarrow B_{p, q}^{\beta}(F, \mu)
$$


such that $\mathcal{R} f=\left.f\right|_{F}$ for $f$ continuous, for $\alpha$ small, $1<p<\infty, 1 \leq q<$ $\infty$, and $\beta=\alpha-\frac{N-d}{p}>0$ (see Theorem 2.6).

This paper is organized as follows. In Section 2, we list all the main results, together with all the definitions needed to state them and some examples where they can be applied, including an example for the trace theorem not covered by the classical results. Sections 3-5 are dedicated to proving these results. In Section 3 we prove the extension theorem in a general setting. For the restriction part, in Section 4 we prove the interpolation result between potential spaces. Finally, in Section 5 we prove the restriction theorems for both potential and Besov functions.

\section{Definitions and statement of the main results}

This section includes all definitions from harmonic analysis on spaces of homogeneous type needed to state the main theorems of the paper, as well as those theorems. It also contains some examples where they can be applied, see Remarks 2.2 and 2.8.

We say $(X, \rho, m)$ is a space of homogeneous type if $\rho$ is a quasi-metric on $X$ and $m$ a measure such that balls and open sets are measurable and that satisfies the doubling property: there exists a constant $C>0$ such that

$$
m\left(B_{\rho}(x, 2 r)\right) \leq C m\left(B_{\rho}(x, r)\right)
$$

for each $x \in X$ and $r>0$.

If $m(\{x\})=0$ for each $x \in X$, by [14] there exists a metric $d$ giving the same topology as $\rho$ and a number $N>0$ such that $(X, d, m)$ satisfies

$$
m\left(B_{d}(x, r)\right) \sim r^{N}
$$

for each $x \in X$ and $0<r<\operatorname{diam}(X)$.

Spaces that satisfy condition (1) are called Ahlfors $N$-regular. Besides $\mathbb{R}^{n}$ (with $N=n$ ), examples include self-similar fractals such as the Cantor ternary set or the Sierpiński gasket.

Throughout this work we use a definition of Besov spaces via a modulus of continuity, equivalent to the classical one for the case of $\mathbb{R}^{n}$, as can be found in [8] (for classical Besov spaces defined this way see for instance [20]). For a definition based on approximations of the identity see $[\mathbf{1 0}]$, and for an equivalence between these and other definitions see $[\mathbf{1 8}]$ and $[\mathbf{9}]$. 
For $1 \leq p<\infty$ and $f \in L_{\mathrm{loc}}^{1}$, its $p$-modulus of continuity is defined as

$$
E_{p} f(t)=\left(\int_{X} f_{B(x, t)}|f(y)-f(x)|^{p} d m(y) d m(x)\right)^{1 / p}
$$

for $t>0$. With this, its Besov norm is defined,

$$
\|f\|_{B_{p, q}^{\alpha}}^{\alpha}=\|f\|_{p}+\left(\int_{0}^{\infty}\left(t^{-\alpha} E_{p} f(t)\right)^{q} \frac{d t}{t}\right)^{1 / q}
$$

(for $1 \leq q<\infty$, and the usual modification for $q=\infty$ ) and then the Besov space $B_{p, q}^{\alpha}$ consists of those functions $f$ with finite norm.

Observe that, as $E_{p} f(t) \leq C\|f\|_{p}$, the integral from 0 to $\infty$ can be restricted to an integral from 0 to 1 , giving equivalent norms. If $m$ is doubling, it can also be discretized as follows:

$$
\|f\|_{B_{p, q}^{\alpha}} \sim\|f\|_{p}+\left(\sum_{k \geq 1}\left(2^{k \alpha} E_{p} f\left(2^{-k}\right)\right)^{q}\right)^{1 / q} .
$$

This definition gives equivalent norms to the ones for $\mathbb{R}^{n}$ and $d$-sets mentioned in the introduction, providing a unified definition for Besov spaces in the 'big set' and the 'small set', unlike [13] where the authors work with different definitions for $\mathbb{R}^{n}$ and $d$-sets.

We can thus state our first main result, which will be proven in Section 3.

Theorem 2.1 (Extension theorem for Besov spaces). Let $(X, d, m)$ be a space of homogeneous type with $d$ a metric, and let $F \subset X$ be closed with $m(F)=0$. Assume $\mu$ is a nontrivial Borel measure with support $F$ which is doubling for balls centered in $F$, and that there exists $\gamma>0$ such that

$$
\frac{m(B)}{\mu(B)} \sim r^{\gamma}
$$

for balls $B$ centered in $F$ with radius $r<2 \operatorname{diam}(F)$.

Then there is an extension operator $\mathcal{E}$ for functions $f \in L_{\mathrm{loc}}^{1}(F, \mu)$ that satisfies, for $1 \leq p<\infty$ and $1 \leq q \leq \infty$ and $0<\beta<1-\gamma / p$,

$$
\mathcal{E}: B_{p, q}^{\beta}(F, \mu) \rightarrow B_{p, q}^{\alpha}(X, m),
$$

where $\alpha=\beta+\gamma / p$.

Certainly, Theorem 2.1 contains the classical result in Theorem 1, Chapter VI from $[\mathbf{1 3}]$ for the case $0<\alpha<1$. 
Remark 2.2. Observe that the quotient condition (2) is weaker than asking for both spaces to be Ahlfors regular. If $X$ satisfies the Ahlfors condition, then clearly so does $F$, but they need not be Ahlfors and still satisfy condition (2). For instance, if $(X, m)$ is a metric measure space satisfying the doubling condition, then $\tilde{X}=X \times \mathbb{R}$ equipped with the product metric and measure will also be doubling (and unbounded), and clearly $F=X \times\{0\}$ and $\tilde{X}$ will satisfy the quotient condition.

As a more interesting example, consider the spaces $\mathbb{R}^{n}$ equipped with the measure $d m=\omega(x) d x_{n}$, where $\omega(x)=|x|^{\alpha}$, and the subspace $F=$ $\mathbb{R}^{d} \times\{0\}, 0<d<n$. Define the measure $d \mu=\omega(x) d x_{d}$ in $F$ and assume $\alpha>-d$. Then clearly both spaces are doubling but neither of them are Ahlfors (except for the trivial case $\alpha=0$ ), however they do satisfy the quotient relation (2) with $\gamma=n-d$.

Remark 2.3. As observed by one of the referees, the quotient condition (2) is related to the $\gamma$-codimensional Hausdorff measure with respect to $m$,

$$
\hat{\mathcal{H}}_{\gamma}(E)=\sup _{\delta} \inf \left\{\sum r_{j}^{-\gamma} m\left(B\left(x_{j}, r_{j}\right)\right): r_{j} \leq \delta, E \subset \bigcup B\left(x_{j}, r_{j}\right)\right\}
$$

(see for instance [16]). For Borel sets $E \subset F$, by the doubling property of $m$ one can go from arbitrary coverings of $E$ to coverings by balls centered in $F$, then by condition (2) one obtains

$$
\mu(E) \sim \hat{\mathcal{H}}_{\gamma}(E)
$$

For the case that $(X, m)$ is Ahlfors $N$-dimensional and $(F, \mu)$ Ahlfors $d$-dimensional with $d<N$ (therefore condition (2) holds for $\gamma=N-d$ ), we actually have that $\mu$ is comparable to the $d$-dimensional Hausdorff measure $\mathcal{H}_{d}$ (see $[\mathbf{2}]$ or $\left.[\mathbf{1 2}]\right)$.

As mentioned in the introduction, to prove the restriction theorem we need to prove first an interpolation result for Potential-type Sobolev spaces.

In $\mathbb{R}^{n}$, Bessel potentials $\mathcal{J}_{\alpha}=(I-\Delta)^{-\alpha / 2}$ (where $\Delta$ is the Laplacian) and its associated Sobolev potential spaces $\mathcal{L}^{\alpha, p}=\mathcal{J}_{\alpha}\left(L^{p}\right)$ play a crucial role in the proof of the restriction theorem in $[\mathbf{1 3}]$.

In Ahlfors spaces with $m(X)=\infty$, Coifman-type approximations of the identity $\left(S_{t}\right)_{t>0}$ can be constructed (see for instance $[\mathbf{7}]$ or $[\mathbf{1 0}]$ ). Potential-type Sobolev spaces are defined and studied in the metric setting in [17] via the kernel

$$
k_{\alpha}(x, y)=\int_{0}^{\infty} \frac{\alpha t^{\alpha}}{\left(1+t^{\alpha}\right)^{2}} s(x, y, t) \frac{d t}{t},
$$


where $s$ is the kernel of the approximation of the identity $\left(S_{t}\right)_{t>0}$ that will be described in Section 4.

With this kernel, the Bessel-type potential is defined as

$$
J_{\alpha} g(x)=\int_{X} k_{\alpha}(x, y) g(y) d m(y),
$$

and turns out to be bounded in $L^{p}$ for $1 \leq p \leq \infty$. The potential space $L^{\alpha, p}=J_{\alpha}\left(L^{p}\right)$ is proved Banach when equipped with the norm

$$
\|f\|_{\alpha, p}=\|f\|_{p}+\inf _{g}\|g\|_{p}
$$

where the infimum is taken over all $g$ with $f=J_{\alpha} g$.

These spaces satisfy, among other things, that continuous functions are dense in $L^{\alpha, p}$ for $\alpha>0$ and $1 \leq p \leq \infty$ (see [17, Corollary 4.6]), and we also have the embedding $L^{\alpha, p} \hookrightarrow B_{p, \infty}^{\alpha}$ for $0<\alpha<1,1 \leq p \leq \infty$. Besides, for $1<p<\infty$ there exists $\alpha_{0}<1$ such that the potential $J_{\alpha}$ is invertible (see Theorem 4.3) and we have the following result, that we will prove in Section 4. This theorem has importance on its own.

Theorem 2.4 (Interpolation theorem for potential spaces). Let $X$ be Ahlfors regular with $m(X)=\infty$. For $1<p, q<\infty, 0<\alpha, \beta<\alpha_{0}$, $0<\theta<1$, and $\gamma=\alpha+\theta(\beta-\alpha)$, we get

$$
B_{p, q}^{\gamma}=\left(L^{\alpha, p}, L^{\beta, p}\right)_{\theta, q} .
$$

For the restriction theorem, we will use the previous result. For that let $(X, d, m)$ be Ahlfors $N$-regular with $m(X)=\infty$ and let $F \subset X$ be a closed subset with $\mu$ a Borel measure supported in $F$ such that $\left(F,\left.d\right|_{F \times F}, \mu\right)$ is Ahlfors $d$-regular, $0<d<N$.

To prove the restriction theorem we first show that potential spaces $L^{\alpha, p}$ leave a trace in $F$, belonging to a certain Besov space. This is done in Section 5 using size and smoothness properties of the kernel $k_{\alpha}$.

Theorem 2.5 (Restriction theorem for potential spaces). Let $X, F$ be as above, and let $0<\alpha<1$ and $1<p<\infty$ satisfying $0<\beta=\alpha-\frac{N-d}{p}<1$. Then there exists a continuous linear operator

$$
\mathcal{R}: L^{\alpha, p}(X, m) \rightarrow B_{p, \infty}^{\beta}(F, \mu)
$$

satisfying $\mathcal{R} f=\left.f\right|_{F}$ for continuous functions in $L^{\alpha, p}$.

Combining Theorem 2.4 with Theorem 2.5, we obtain the result we were looking for. 
Theorem 2.6 (Restriction theorem for Besov spaces). Under the conditions of Theorem 2.5, if $\alpha<\alpha_{0}, 1<p<\infty$, and $\beta=\alpha-\frac{N-d}{p}>0$, then for $1 \leq q<\infty$ there exists a continuous linear operator

$$
\mathcal{R}: B_{p, q}^{\alpha}(X, m) \rightarrow B_{p, q}^{\beta}(F, \mu)
$$

such that $\mathcal{R} f=\left.f\right|_{F}$ for $f$ continuous in $B_{p, q}^{\alpha}(X, m)$.

With the previous results, we can now state the full version of the trace theorem. In the context of Theorem 2.6, the 'big set' $X$ is Ahlfors $N$-dimensional and the 'small set' $F$ is $d$-dimensional with $0<d<N$, and they clearly satisfy the quotient property (2) for $\gamma=N-d$.

Thus the conditions of both theorems can be met, as long as we restrict the regularity for orders $0<\alpha<\alpha_{0}$, where $\alpha_{0}$ comes from Theorem 4.3.

Theorem 2.7 (Trace theorem for Besov spaces). Let $(X, d, m)$ be an Ahlfors $N$-regular space with $m(X)=\infty$, and $F \subset X$ a closed subset with $m(F)=0$ such that there exists a measure $\mu$ for which $\left(F,\left.d\right|_{F \times F}, \mu\right)$ is Ahlfors d-regular with $0<d<N$. Then for $0<\beta<\alpha<\alpha_{0}$, $1<p<\infty$, and $1 \leq q<\infty$ satisfying $\beta=\alpha-\frac{N-d}{p}$, we have that

$$
\left.B_{p, q}^{\alpha}(X, m)\right|_{F}=B_{p, q}^{\beta}(F, \mu) .
$$

That is, there exist continuous linear operators $\mathcal{E}: B_{p, q}^{\beta}(F, \mu) \rightarrow B_{p, q}^{\alpha}(X, m)$ and $\mathcal{R}: B_{p, q}^{\alpha}(X, m) \rightarrow B_{p, q}^{\beta}(F, \mu)$ satisfying

$$
f(t)=\lim _{r \rightarrow 0} f_{B(t, r)} \mathcal{E} f d m
$$

for $\mu$-almost every $t \in F, f \in B_{p, q}^{\beta}(F, \mu)$, and

$$
\mathcal{R} \mathbf{f}=\left.\mathbf{f}\right|_{F}
$$

for every $\mathbf{f} \in B_{p, q}^{\alpha}(X, m)$ continuous. In particular, $\mathcal{R E} f=f$ for all $f \in B_{p, q}^{\beta}(F, \mu)$.

Remark 2.8. When $X=\mathbb{R}^{n}$, Theorem 4.3 can be replaced with a more classical one (see for instance [20]), and thus we have $\alpha_{0}=1$ in Theorem 2.7, recovering the classical result from $[\mathbf{1 3}]$.

As an additional example, we can obtain an Ahlfors regular space satisfying $m(X)=\infty$ modifying the Sierpiński gasket $T$ by taking dilations (powers of 2): $\tilde{T}=\cup_{k \geq 0} 2^{k} T$. This $\tilde{T}$ preserves some properties of the original triangle, including the Ahlfors character with $N=\frac{\log 3}{\log 2}$. 


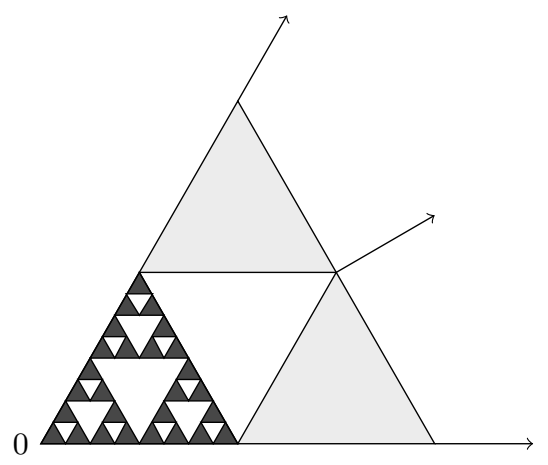

Figure 1. The modified Sierpiński gasket $\tilde{T}$.

It is clear that the real half-line $R^{+}=[0, \infty) \times\{0\}$ is a closed subset of $\tilde{T}$, and it is Ahlfors regular with $d=1$. Therefore we obtain as a new result that

$$
\left.B_{p, q}^{\alpha}(\tilde{T})\right|_{R^{+}}=B_{p, q}^{\beta}\left(R^{+}\right)
$$

for $0<\alpha<\alpha_{0}(\tilde{T})$ and $0<\beta=\alpha-\frac{N-d}{p}$ (observe that this case is not included in the classical theorem from $[\mathbf{1 3}]$, as clearly $\tilde{T} \neq \mathbb{R}^{n}$ ).

\section{Proof of the extension theorem}

An essential tool in proving the extension theorem is a Whitney-type covering lemma that remains valid in the metric setting. The one we will use is an adaptation of a theorem found in [1], its proof can also be found in [5] or [15]. As this result is purely geometrical, it remains valid in a much general scenario. As the doubling property implies the weak homogeneity property, the result holds in our case.

Lemma 3.1 (Whitney type covering and partition of unity). Let $F$ be a closed subset of a metric space $(X, d)$ that satisfies the weak homogeneity property. Then there exists a (countable) collection $\left\{B_{i}=B\left(x_{i}, r_{i}\right)\right\}_{i}$ of balls satisfying

1. $\left\{B_{i}\right\}$ are pairwise disjoint;

2. $0<d\left(x_{i}, F\right)<\frac{1}{2}$ for each $i$ and $\{x: 0<d(x, F)<1\} \subset \cup_{i} 3 B_{i}$;

3. $6 r_{i} \leq d(x, F) \leq 18 r_{i}$ for each $x \in 6 B_{i}$, for each $i$;

4. for each $i$ there exists $t_{i} \in F$ satisfying $d\left(x_{i}, t_{i}\right)<18 r_{i}$;

5. if $\Omega=\cup_{i} 6 B_{i}$, then $F \cap \Omega=\emptyset$ and $\partial F \subset \bar{\Omega}$;

6. there exists $M>0$ such that for each $i, \#\left\{j: 6 B_{i} \cap 6 B_{j} \neq \emptyset\right\} \leq M$. 
Furthermore, there exists a collection $\left\{\varphi_{i}\right\}_{i}$ of real functions satisfying

1. $3 B_{i} \subset \operatorname{supp} \varphi_{i} \subset 6 B_{i}$;

2. $0 \leq \varphi_{i} \leq 1$ and $0 \leq \sum_{i} \varphi_{i} \leq 1$;

3. $\sum_{i} \varphi_{i}(x)=1$ if $0<d(x, F)<1$;

4. $\sum_{i} \varphi_{i} \equiv 0$ outside of $\Omega$;

5. $\varphi_{i} \equiv 1$ in $B_{i}$;

6. for each $i,\left|\varphi_{i}(x)-\varphi_{i}(y)\right| \leq \frac{C}{r_{i}} d(x, y)$ with $C$ independent of $i$.

We will also need the following result.

Lemma 3.2 (Bounded overlap). Let $(X, d)$ be a metric space with the weak homogeneity property and let $1 \leq a<b, \kappa>1$. There exists a constant $C$ such that, if $\left\{B_{i}=B\left(x_{i}, r_{i}\right)\right\}_{i}$ is a family of disjoint balls, and $r>0$,

$$
\sum_{i: a r \leq r_{i} \leq b r} \chi_{\kappa B_{i}} \leq C .
$$

Proof of Theorem 2.1: Let us first define the extension operator: let us take $\left\{B_{i}, \varphi_{i}\right\}_{i}$ as in Whitney's Lemma (Lemma 3.1). If $f \in L_{\text {loc }}^{1}(F, \mu)$ and $x \in X$, we define

$$
\mathcal{E} f(x)=\sum_{i} \varphi_{i}(x) f_{19 B_{i}} f d \mu .
$$

Observe that by the properties possessed by $B_{i}$ and $\varphi_{i}, \mu\left(19 B_{i}\right)>0$ for each $i$ so the constants multiplying each $\varphi_{i}$ make sense. Also, $\mathcal{E} f$ is continuous in $\Omega=\cup_{i} 6 B_{i}$ and $\mathcal{E} f \equiv 0$ in the exterior of $\Omega$, and it will not necessarily be continuous in $F \subset \partial \Omega$.

We will prove the theorem in three parts: we first check that $\mathcal{E}$ is in fact an extension operator for each $f \in L_{\text {loc }}^{1}(F, \mu)$. Next we prove $\mathcal{E}$ is bounded from $L^{p}(F, \mu)$ to $L^{p}(X, m)$, and we finish the theorem proving $\mathcal{E}: B_{p, q}^{\beta}(F, \mu) \rightarrow B_{p, q}^{\alpha}(X, m)$ for $\alpha, \beta$ as in the statement of the theorem.

We first need to see that $\left.\mathcal{E} f\right|_{F}=f$ (remember $m(F)=0$ and that $F \subset \partial \Omega$ ). As mentioned in the introduction, this means that we have to prove that the function

$$
\left.\mathcal{E} f\right|_{F}\left(t_{0}\right)=\lim _{r \rightarrow 0} f_{B\left(t_{0}, r\right)} \mathcal{E} f(x) d m(x)
$$

is well defined and equals $f\left(t_{0}\right)$ for $\mu$-almost every $t_{0} \in F$.

As $\mu$ is doubling and $f \in L_{\text {loc }}^{1}(F, \mu)$, Lebesgue's differentiation theorem holds. Take $t_{0} \in F$ a $\mu$-Lebesgue point of $f$ and $0<r<1$. For $x$ 
with $0<d(x, F)<1$ we have $\sum_{i} \varphi_{i}(x)=1$, so

$$
\mathcal{E} f(x)-f\left(t_{0}\right)=\sum_{i} \varphi_{i}(x) f_{19 B_{i}}\left(f(t)-f\left(t_{0}\right)\right) d \mu(t),
$$

and then

$$
\left|\mathcal{E} f(x)-f\left(t_{0}\right)\right| \leq \sum_{i} \chi_{6 B_{i}}(x) f_{19 B_{i}}\left|f(t)-f\left(t_{0}\right)\right| d \mu(t) .
$$

Let now $x \in B\left(t_{0}, r\right) \backslash F$, then there exists $k$ such that $2^{-k}<c r$ and $d(x, F) \sim 2^{-k}$, as $t_{0} \in F$, and in that case

$$
\begin{aligned}
\left|\mathcal{E} f(x)-f\left(t_{0}\right)\right| & \leq \int_{F}\left|f(t)-f\left(t_{0}\right)\right|\left(\sum_{r_{i} \sim 2^{-k}} \chi_{19 B_{i}}(t) \frac{\chi_{6 B_{i}}(x)}{\mu\left(19 B_{i}\right)}\right) d \mu(t) \\
& \leq \int_{B\left(t_{0}, r+c 2^{-k}\right)}\left|f(t)-f\left(t_{0}\right)\right|\left(\sum_{r_{i} \sim 2^{-k}} \chi_{19 B_{i}}(t) \frac{\chi_{19 B_{i}}(x)}{\mu\left(19 B_{i}\right)}\right) d \mu(t) \\
& \leq \int_{B\left(t_{0}, c r\right)}\left|f(t)-f\left(t_{0}\right)\right|\left(\sum_{r_{i} \sim 2^{-k}} \chi_{19 B_{i}}(t) \frac{\chi_{19 B_{i}}(x)}{\mu\left(19 B_{i}\right)}\right) d \mu(t) ;
\end{aligned}
$$

now integrating over $B\left(t_{0}, r\right)$ (using $m\left(B\left(t_{0}, r\right) \cap F\right)=0$ ), by the bounded overlap property and the relation between the measures,

$$
\begin{aligned}
\int_{B\left(t_{0}, r\right)}\left|\mathcal{E} f(x)-f\left(t_{0}\right)\right| d m(x) \\
\leq \sum_{2^{-k} \leq c r} \int_{x \in B\left(t_{0}, r\right), d(x, F) \sim 2^{-k}}\left|\mathcal{E} f(x)-f\left(t_{0}\right)\right| d m(x) \\
\leq \sum_{2^{-k} \leq c r} \int_{B\left(t_{0}, c r\right)}\left|f(t)-f\left(t_{0}\right)\right|\left(\sum_{r_{i} \sim 2^{-k}} \chi_{19 B_{i}}(t) \frac{m\left(19 B_{i}\right)}{\mu\left(19 B_{i}\right)}\right) d \mu(t) \\
\leq C\left(\sum_{2^{-k} \leq c r} 2^{-k \gamma}\right) \int_{B\left(t_{0}, c r\right)}\left|f(t)-f\left(t_{0}\right)\right| d \mu(t) \\
\leq C r^{\gamma} \int_{B\left(t_{0}, c r\right)}\left|f(t)-f\left(t_{0}\right)\right| d \mu(t) \\
\leq C \frac{m\left(B\left(t_{0}, c r\right)\right)}{\mu\left(B\left(t_{0}, c r\right)\right)} \int_{B\left(t_{0}, c r\right)}\left|f(t)-f\left(t_{0}\right)\right| d \mu(t) .
\end{aligned}
$$


In other words,

$$
f_{B\left(t_{0}, r\right)}\left|\mathcal{E} f(x)-f\left(t_{0}\right)\right| d m(x) \leq C f_{B\left(t_{0}, c r\right)}\left|f(t)-f\left(t_{0}\right)\right| d \mu(t),
$$

and as the right side tends to 0 when $r \rightarrow 0$ for $\mu$-a.e. $t_{0} \in F$, the left side also tends to 0 and $\left.\mathcal{E} f\right|_{F}=f \mu$-a.e.

We need to check now that there exists $C>0$ such that $\|\mathcal{E} f\|_{p, m} \leq$ $C\|f\|_{p, \mu}$ for every $f \in L^{p}(F, \mu)$.

We have

$|\mathcal{E} f(x)|=\left|\sum_{i} \varphi_{i}(x) f_{19 B_{i}} f d \mu\right| \leq \sum_{i} \varphi_{i}(x) \chi_{6 B_{i}}(x)\left(f_{19 B_{i}}|f|^{p} d \mu\right)^{1 / p}$,

and so by the discrete version of Hölder's inequality, as $\left(\sum_{i} \varphi_{i}(x)^{p^{\prime}}\right)^{1 / p^{\prime}} \leq$ 1 ,

$$
\begin{aligned}
|\mathcal{E} f(x)|^{p} & \leq \sum_{i} \chi_{6 B_{i}}(x) f_{19 B_{i}}|f|^{p} d \mu \\
& \leq \int_{F}|f(t)|^{p}\left(\sum_{i} \chi_{19 B_{i}}(t) \frac{\chi_{19 B_{i}}(x)}{\mu\left(19 B_{i}\right)}\right) d \mu(t) .
\end{aligned}
$$

Once again by bounded overlap,

$$
\begin{aligned}
\int_{X}|\mathcal{E} f(x)|^{p} d m(x) & \leq C \int_{F}|f(t)|^{p}\left(\sum_{i} \chi_{19 B_{i}}(t) \frac{m\left(19 B_{i}\right)}{\mu\left(19 B_{i}\right)}\right) d \mu(t) \\
& \leq C \int_{F}|f(t)|^{p}\left(\sum_{k} \sum_{r_{i} \sim 2^{-k}} \chi_{19 B_{i}}(t) \frac{m\left(19 B_{i}\right)}{\mu\left(19 B_{i}\right)}\right) d \mu(t) \\
& \leq C \int_{F}|f(t)|^{p}\left(\sum_{k} 2^{-k \gamma}\right) d \mu(t) \leq C\|f\|_{p, \mu}^{p} .
\end{aligned}
$$

Observe that the quotient relation of measures (2) is essential in this proof. In the classical case, this is just the power $r^{n-d}$, where $r$ is the radius of a ball. The rest of the proof follows a similar pattern to that in $[\mathbf{1 3}]$, taking into account this important difference, so we will leave it out.

\section{Proof of the interpolation theorem}

In this section we will prove Theorem 2.4, that is, under certain conditions, the interpolated space $\left(L^{\alpha, p}, L^{\beta, p}\right)_{\theta, q}$, where $L^{\alpha, p}$ and $L^{\beta, p}$ are 
the potential spaces described in Section 2 and defined in [17], coincides with the Besov space $B_{p, q}^{\gamma}$, with $\gamma=\alpha+\theta(\beta-\alpha)$.

The following results and definitions, concerning interpolation between Banach spaces, are based on $[\mathbf{3}]$.

Given $X, Y$ Banach, both embedded in the same topological vector space, Peetre's $K$ and $J$-functionals for these spaces $X, Y$ are defined as

$$
\begin{gathered}
K f(t)=\inf _{f=g+h}\|g\|_{X}+t\|h\|_{Y}, \quad f \in X+Y ; \\
J f(t)=\max \left(\|f\|_{X}, t\|f\|_{Y}\right), \quad f \in X \cap Y .
\end{gathered}
$$

Now, for $0<\theta<1,1 \leq q \leq \infty$, the interpolated space $(X, Y)_{\theta, q}$ is defined as the set of those $f$ with the following finite norm

$$
\|f\|_{\theta, q, K}=\left\|t^{-\theta} K f(t)\right\|_{L^{q}\left((0, \infty), \frac{d t}{t}\right)} .
$$

One can also define the same interpolated space with the aid of the $J$-functional, as the set of those $f \in X+Y$ that have a Bochner integral decomposition

$$
f=\int_{0}^{\infty} A_{s} f \frac{d s}{s},
$$

with $A_{s} f \in X \cap Y$ for each $s$, such that

$$
\|f\|_{\theta, q, J}=\inf _{A_{s}}\left\|s^{-\theta} J\left(A_{s} f\right)(s)\right\|_{L^{q}\left((0, \infty), \frac{d s}{s}\right)},
$$

where the infimum is taken over all possible decompositions of $f$.

Proof of Theorem 2.4: Let $(X, d, m)$ be Ahlfors $N$-regular with $m(X)=$ $\infty$. We aim to show $\left(L^{\alpha, p}, L^{\beta, p}\right)_{\theta, q}=B_{p, q}^{\gamma}$ by proving two (continuous) inclusions: first we see that

$$
\left(L^{\alpha, p}, L^{\beta, p}\right)_{\theta, q} \hookrightarrow B_{p, q}^{\gamma},
$$

for $0<\alpha, \beta<1,1 \leq p \leq \infty, 1 \leq q<\infty$ satisfying the relation mentioned above, using the $K$ functional described above. This will be done in Lemma 4.1.

To check the other inclusion

$$
B_{p, q}^{\gamma} \hookrightarrow\left(L^{\alpha, p}, L^{\beta, p}\right)_{\theta, q},
$$

we prove

$$
\|f\|_{\theta, q, J} \leq C\|f\|_{B_{p, q}^{\gamma}},
$$

where $J$ is the $J$-functional (also described above), for the case $0<\alpha, \beta<$ $\alpha_{0}$ and $1<p, q<\infty$. This result will be proven in Theorem 4.4. 
Lemma 4.1. For $0<\alpha, \beta<1,1 \leq p, q \leq \infty, 0<\theta<1$, and $\gamma=$ $(1-\theta) \alpha+\theta \beta$,

$$
\left(L^{\alpha, p}, L^{\beta, p}\right)_{\theta, q} \hookrightarrow B_{p, q}^{\gamma} .
$$

Proof: The embedding $\left(L^{\alpha, p}, L^{\beta, p}\right)_{\theta, q} \hookrightarrow B_{p, q}^{\gamma}$ is a consequence of the fact that $L^{\alpha, p} \hookrightarrow B_{p, \infty}^{\alpha}$ for $0<\alpha<1$ (see [17], and the same holds for $\beta$ ), as mentioned in Section 2, so by interpolation

$$
\left(L^{\alpha, p}, L^{\beta, p}\right)_{\theta, q} \hookrightarrow\left(B_{p, \infty}^{\alpha}, B_{p, \infty}^{\beta}\right)_{\theta, q} .
$$

Then the result follows from the fact that

$$
\left(B_{p, \infty}^{\alpha}, B_{p, \infty}^{\beta}\right)_{\theta, q} \hookrightarrow B_{p, q}^{\gamma} .
$$

We omit its proof, as the ideas coincide with the proof of Theorem 5.6.1 in [4], except that theorem is done in the discrete setting.

To prove the other embedding, we follow the ideas in $[\mathbf{1 9}]$ for $\mathbb{R}^{n}$ but in the continuous case (instead of the discrete one), where J. Peetre gives an adequate decomposition of functions in $B_{p, q}^{\gamma}$ in terms of functions in $\mathcal{L}^{\alpha, p} \cap \mathcal{L}^{\beta, p}$, and each piece satisfies certain bounds in the norms of $\mathcal{L}^{\alpha, p}$ and $\mathcal{L}^{\beta, p}$. This is done in this classical case using the fact that the Bessel potential $\mathcal{J}_{\alpha}$ is invertible and $\mathcal{J}_{\alpha}^{-1} \approx I+\mathcal{D}_{\alpha}$ (and the same for $\beta$ ). As it will be mentioned in Theorem 4.3, this same result holds in Ahlfors spaces for the case $0<\alpha, \beta<\alpha_{0}$ and $1<p<\infty$.

For this, we need the following results from harmonic analysis in the metric setting.

In Ahlfors $N$-regular spaces, the following version of the $T 1$ Theorem hold (see for instance [6], or [7] for the case $N=1$ ). We require $m(X)=$ $\infty$.

A continuous function $K: X \times X \backslash \Delta \rightarrow \mathbb{R}$ (where $\Delta=\{(x, x): x \in$ $X\})$ is a standard kernel if there exist constants $0<\eta \leq 1, C>0$ such that

- $|K(x, y)| \leq C d(x, y)^{-N}$;

- for $x \neq y, d\left(x, x^{\prime}\right) \leq c d(x, y)$ (with $c<1$ ) we have

$$
\left|K(x, y)-K\left(x^{\prime}, y\right)\right| \leq C d\left(x, x^{\prime}\right)^{\eta} d(x, y)^{-(N+\eta)} ;
$$

- for $x \neq y, d\left(y, y^{\prime}\right) \leq c d(x, y)$ (with $c<1$ ) we have

$$
\left|K(x, y)-K\left(x, y^{\prime}\right)\right| \leq C d\left(y, y^{\prime}\right)^{\eta} d(x, y)^{-(N+\eta)} .
$$


Let $C_{c}^{\gamma}$ denote the space of Lipschitz- $\gamma$ functions with compact support. A linear continuous operator $T: C_{c}^{\gamma} \rightarrow\left(C_{c}^{\gamma}\right)^{\prime}$ for $0<\gamma \leq 1$ is a singular integral operator with associated standard kernel $K$ if it satisfies

$$
\langle T f, g\rangle=\iint K(x, y) f(y) g(x) d m(y) d m(x),
$$

for $f, g \in C_{c}^{\gamma}$ with disjoint supports. If a singular integral operator can be extended to a bounded operator on $L^{2}$ it is called a Calderón-Zygmund operator or CZO.

Every CZO is bounded in $L^{p}$ for $1<p<\infty$. We say that an operator is weakly bounded if

$$
|\langle T f, g\rangle| \leq C m(B)^{1+2 \gamma / N}[f]_{\gamma}[g]_{\gamma},
$$

for $f, g \in C_{c}^{\gamma}(B)$, for each ball $B$.

Theorem 4.2 ( $T 1$ Theorem). Let $T$ be a singular integral operator. Then $T$ is a CZO if $T 1=T^{*} 1=0$ and $T$ is weakly bounded.

In this setting, Coifman-type approximations of the identity $\left(S_{t}\right)_{t>0}$ can be constructed (see for instance $[\mathbf{7}]$ or $[\mathbf{1 0}]$ ). Let $s$ be the kernel associated to $\left(S_{t}\right)$, meaning

$$
S_{t} f(x)=\int_{X} f(y) s(x, y, t) d m(y)
$$

and define

$$
q(x, y, t)=-t \frac{d}{d t} s(x, y, t), \quad Q_{t} f(x)=\int_{X} f(y) q(x, y, t) d m(y) .
$$

This satisfies the following:

Q1. $Q_{t} 1 \equiv 0$ for all $t>0$;

Q2. $q(x, y, t)=q(y, x, t)$ for $x, y \in X, t>0$;

Q3. $|q(x, y, t)| \leq C / t^{N}$ for $x, y \in X, t>0$;

Q4. $q(x, y, t)=0$ if $d(x, y)>4 t$;

Q5. $\left|q(x, y, t)-q\left(x^{\prime}, y, t\right)\right| \leq C^{\prime} \frac{1}{t^{N+1}} d\left(x, x^{\prime}\right)$;

Q6. $Q_{t}: L^{p} \rightarrow L^{p}$.

In this case, the following version of Calderón's reproducing formula holds. There exists a kernel $\tilde{q}$ (related to the kernel $q$ ) such that

$$
f=\int_{0}^{\infty} \tilde{Q}_{s} Q_{s} f \frac{d s}{s}
$$

for $\tilde{Q}_{s} f=\int_{X} \tilde{q}(\cdot, y, t) f(y) d m(y)$. This $\tilde{q}$ satisfies, for any $0<\xi<1$, 
Qิ1. $|\tilde{q}(x, y, t)| \leq C \frac{t^{\xi}}{(t+d(x, y))^{N+\xi}}$;

Q22. $\int_{X} \tilde{q}(x, y, t) d m(y)=\int_{X} \tilde{q}(z, x, t) d m(z)=0$ for each $x$ and $t>0$;

Q̄3. for $d\left(x, x^{\prime}\right)<C(t+d(x, y))$,

$$
\left|\tilde{q}(x, y, t)-\tilde{q}\left(x^{\prime}, y, t\right)\right| \leq C \frac{t^{\xi} d\left(x, x^{\prime}\right)^{\xi}}{(t+d(x, y))^{N+2 \xi}} .
$$

See for instance $[\mathbf{1 0}]$ for a discrete version.

With the fractional derivative $D_{\alpha}$ defined as

$$
D_{\alpha} f(x)=\int_{0}^{\infty} t^{-\alpha} Q_{t} f(x) \frac{d t}{t}
$$

(see for instance $[\mathbf{7}]$ or $[\mathbf{1 7}]$ ), there exists $0<\alpha_{0}<1$ such that the following theorem holds.

Theorem 4.3 (see $\left[\mathbf{1 7}\right.$, Theorem 6.1]). For $1<p<\infty$ and $0<\alpha<\alpha_{0}$, we have

$$
f \in L^{\alpha, p} \text { if and only if } f, D_{\alpha} f \in L^{p} .
$$

Furthermore,

$$
\|f\|_{\alpha, p} \sim\left\|\left(I+D_{\alpha}\right) f\right\|_{p}
$$

Now we are ready to prove the embedding.

Theorem 4.4. For $1<p, q<\infty, 0<\alpha, \beta<\alpha_{0}, 0<\theta<1$, and $\gamma=\alpha+\theta(\beta-\alpha)$, we get

$$
B_{p, q}^{\gamma} \hookrightarrow\left(L^{\alpha, p}, L^{\beta, p}\right)_{\theta, q} .
$$

Proof: Assume there exists a decomposition of the identity in terms of operators $\left(A_{t}\right)_{t>0}$ for functions $f \in B_{p, q}^{\gamma}$ as $A_{t}(f) \in L^{\alpha, p} \cap L^{\beta, p}$ that is

$$
f=\int_{0}^{\infty} A_{t}(f) \frac{d t}{t}=\int_{0}^{1} A_{t}(f) \frac{d t}{t}+A f
$$

satisfying

$$
\begin{gathered}
\left\|A_{t}(f)\right\|_{\alpha, p} \leq C t^{-\alpha} E_{p} f(t), \quad 0<t<1 ; \\
\|A f\|_{\alpha, p} \leq C\|f\|_{p} ;
\end{gathered}
$$

and similar bounds for $\beta$. The existence of such decomposition will be proved separately in Lemma 4.5 .

Under these conditions, we will see that

$$
B_{p, q}^{\gamma} \hookrightarrow\left(L^{\alpha, p}, L^{\beta, p}\right)_{\theta, q} .
$$

By definition of the $J$-functional:

$$
J\left(A_{t}(f)\right)(s)=\max \left(\left\|A_{t}(f)\right\|_{\alpha, p}, s\left\|A_{t}(f)\right\|_{\beta, p}\right),
$$


then by hypothesis

$$
J\left(A_{t}(f)\right)\left(t^{\beta-\alpha}\right) \leq C \max \left(t^{-\alpha} E_{p} f(t), t^{\beta-\alpha} t^{-\beta} E_{p} f(t)\right)=C t^{-\alpha} E_{p} f(t) .
$$

Also,

$$
J(A f)(1)=\max \left(\|A f\|_{\alpha, p},\|A f\|_{\beta, p}\right) \leq C\|f\|_{p} .
$$

Now, assume $\alpha<\beta$ and observe

$$
f=A f+\int_{0}^{1} \frac{1}{\beta-\alpha} A_{t^{1 /(\beta-\alpha)}}(f) \frac{d t}{t}=A f+\int_{0}^{1} \tilde{A}_{t}(f) \frac{d t}{t},
$$

therefore

$$
\begin{aligned}
\|f\|_{\theta, q, J} & \leq J(A f)(1)+\left(\int_{0}^{1} t^{-\theta(\beta-\alpha) q} J\left(\tilde{A}_{t^{\beta-\alpha}}(f)\right)\left(t^{\beta-\alpha}\right)^{q} \frac{d t}{t}\right)^{1 / q} \\
& \leq J(A f)(1)+C\left(\int_{0}^{\infty} t^{-\theta(\beta-\alpha) q} J\left(A_{t}(f)\right)\left(t^{\beta-\alpha}\right)^{q} \frac{d t}{t}\right)^{1 / q} \\
& \leq C\|f\|_{p}+C\left(\int_{0}^{\infty} t^{-\gamma q} E_{p} f(t)^{q} \frac{d t}{t}\right)^{1 / q} \leq C\|f\|_{B_{p, q}^{\gamma}} .
\end{aligned}
$$

We will show in the next lemma that, under the assumption $\alpha, \beta<\alpha_{0}$, Calderón's reproducing formula

$$
f=\int_{0}^{\infty} \tilde{Q}_{t} Q_{t} f \frac{d t}{t}=\int_{0}^{1} \tilde{Q}_{t} Q_{t} f \frac{d t}{t}+\mathbf{Q} f
$$

gives the decomposition we want, for $A_{t}=\tilde{Q}_{t} Q_{t}$ and $A=\mathbf{Q}$, using the fact that $\|g\|_{\alpha, p} \sim\left\|\left(I+D_{\alpha}\right) g\right\|_{p}$ for $\alpha<\alpha_{0}$ (see Theorem 4.3).

Observe that this decomposition is very different from the classical one found in $[\mathbf{1 9}]$, even though it is a version of Calderón's reproducing formula.

Lemma 4.5. For $1<p<\infty, 0<\alpha<1$, and $0<t<1$ we have

$$
\left\|\left(I+D_{\alpha}\right) \tilde{Q}_{t} Q_{t} f\right\|_{p} \leq C t^{-\alpha} E_{p} f(c t) ; \quad\left\|\left(I+D_{\alpha}\right) \mathbf{Q} f\right\|_{p} \leq C\|f\|_{p} .
$$

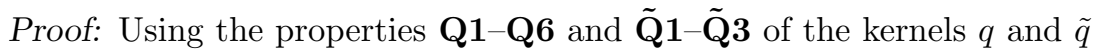
and routine calculations, it is not hard to see that, for $1<p<\infty$, $0<\alpha<1$, and $s>0$,

$$
\left\|D_{\alpha} \tilde{Q}_{s} Q_{s} f\right\|_{p} \leq C s^{-\alpha}\left\|Q_{s} f\right\|_{p}
$$


From this result, and the fact that $\left\|Q_{t} f\right\|_{p} \leq C E_{p} f(c t)$ and that $\tilde{Q}_{t}$ is bounded in $L^{p}$, we obtain

$$
\begin{aligned}
\left\|\left(I+D_{\alpha}\right) \tilde{Q}_{t} Q_{t} f\right\|_{p} & \leq\left\|\tilde{Q}_{t} Q_{t} f\right\|_{p}+C t^{-\alpha} E_{p} f(c t) \\
& \leq C E_{p} f(c t)+C t^{-\alpha} E_{p} f(c t) \\
& \leq C t^{-\alpha} E_{p} f(c t) .
\end{aligned}
$$

The main part of the proof is to show that for $1<p<\infty$,

$$
\|\mathbf{Q} f\|_{p} \leq C\|f\|_{p}
$$

Once we obtain this, the theorem follows, as

$$
\begin{aligned}
\left\|\left(I+D_{\alpha}\right) \mathbf{Q} f\right\|_{p} & \leq C\|f\|_{p}+\int_{1}^{\infty}\left\|D_{\alpha} \tilde{Q}_{t} Q_{t} f\right\|_{p} \frac{d t}{t} \\
& \leq C\|f\|_{p}+C \int_{1}^{\infty} t^{-\alpha}\left\|Q_{t} f\right\|_{p} \frac{d t}{t} \\
& \leq C\|f\|_{p}+C\|f\|_{p} \int_{1}^{\infty} t^{-\alpha} \frac{d t}{t} \\
& \leq C\|f\|_{p},
\end{aligned}
$$

where we have used Minkowski's integral inequality, inequality (3), and the fact that $Q_{t}$ is bounded in $L^{p}$.

We have

$$
\int_{1}^{\infty} \tilde{Q}_{t} Q_{t} f(x) \frac{d t}{t}=\int_{X}\left(\int_{1}^{\infty} \int_{X} \tilde{q}(x, y, t) q(y, z, t) d m(y) \frac{d t}{t}\right) f(z) d m(z),
$$

then if we prove that

$$
\mathbf{Q}(x, z)=\int_{1}^{\infty} \int_{X} \tilde{q}(x, y, t) q(y, z, t) d m(y) \frac{d t}{t}
$$

is a standard kernel and the $T 1$ Theorem holds for the operator $\mathbf{Q}$, we will get our result. The following results make use of properties Q3, Q4, Q5 and $\tilde{\mathbf{Q}} 1, \tilde{\mathbf{Q}} 2, \tilde{\mathbf{Q}} 3$ of the kernels $q$ and $\tilde{q}$. 
- Size of the kernel Q: as $d(y, z)<c t<c(t+d(x, z))$ where $q(y, z, t) \neq 0$,

$$
\begin{aligned}
|\mathbf{Q}(x, z)| & \leq\left|\int_{1}^{\infty} \int_{X}(\tilde{q}(x, y, t)-\tilde{q}(x, z, t)) q(y, z, t) d m(y) \frac{d t}{t}\right| \\
& \leq C \int_{1}^{\infty} \int_{d(y, z)<c t} \frac{t^{\xi} d(y, z)^{\xi}}{(t+d(x, z))^{N+2 \xi}} \frac{1}{t^{N}} d m(y) \frac{d t}{t} \\
& \leq C \int_{1}^{\infty} \frac{t^{2 \xi}}{(t+d(x, z))^{N+2 \xi}} \frac{d t}{t} \\
& \leq C \frac{1}{d(x, z)^{N+2 \xi}} \int_{1}^{d(x, z)} t^{2 \xi} \frac{d t}{t}+C \int_{d(x, z)}^{\infty} \frac{1}{t^{N}} \frac{d t}{t} \\
& \leq C \frac{1}{d(x, z)^{N} .}
\end{aligned}
$$

- Regularity (1): for $d\left(x, x^{\prime}\right)<C d(x, z)$, then $d\left(x, x^{\prime}\right)<C d(x, z) \leq$ $C(d(x, y)+d(y, z)) \leq C(t+d(x, y))$,

$$
\begin{aligned}
& \left|\mathbf{Q}(x, z)-\mathbf{Q}\left(x^{\prime}, z\right)\right| \leq \int_{1}^{\infty} \int_{d(y, z)<4 t}\left|\tilde{q}(x, y, t)-\tilde{q}\left(x^{\prime}, y, t\right)\right| \frac{1}{t^{N}} d m(y) \frac{d t}{t} \\
& \quad \leq C d\left(x, x^{\prime}\right)^{\xi} \int_{1}^{\infty} \frac{1}{t^{N-\xi}} \int_{d(y, z)<4 t} \frac{1}{(t+d(x, y))^{N+2 \xi}} d m(y) \frac{d t}{t} \\
& \quad \leq \int_{0}^{d(x, z) / 5}+\int_{d(x, z) / 5}^{\infty}=I+I I .
\end{aligned}
$$

For $I$, as $d(x, z)>5 t>4 t>d(y, z)$, we have $d(x, z)>\frac{5}{4} d(y, z)$ and therefore $d(x, y)>\frac{1}{5} d(x, z)$, so

$$
I \leq C \frac{d\left(x, x^{\prime}\right)^{\xi}}{d(x, z)^{N+2 \xi}} \int_{0}^{d(x, z) / 5} \frac{1}{t^{N-\xi}} t^{N} \frac{d t}{t} \leq C \frac{d\left(x, x^{\prime}\right)^{\xi}}{d(x, z)^{N+\xi}}
$$

and for $I I$,

$$
I I \leq C d\left(x, x^{\prime}\right)^{\xi} \int_{d(x, z) / 5}^{\infty} \frac{1}{t^{N-\xi}} \frac{t^{N}}{t^{N+2 \xi}} \frac{d t}{t} \leq C \frac{d\left(x, x^{\prime}\right)^{\xi}}{d(x, z)^{N+\xi}}
$$


- Regularity (2): if $d\left(z, z^{\prime}\right) \leq C d(x, z)$, then

$$
\begin{aligned}
\left|\mathbf{Q}(x, z)-\mathbf{Q}\left(x, z^{\prime}\right)\right|=\left|\int_{1}^{\infty} \int_{X} \tilde{q}(x, y, t)\left(q(y, z, t)-q\left(y, z^{\prime}, t\right)\right) d m(y) \frac{d t}{t}\right| \\
\leq \int_{d\left(z, z^{\prime}\right) / C}^{\infty}\left|\int_{X}(\tilde{q}(x, y, t)-\tilde{q}(x, z, t))\left(q(y, z, t)-q\left(y, z^{\prime}, t\right)\right) d m(y)\right| \frac{d t}{t} \\
\quad+\int_{0}^{d\left(z, z^{\prime}\right) / C}\left|\int_{X}(\tilde{q}(x, y, t)-\tilde{q}(x, z, t)) q(y, z, t) d m(y)\right| \frac{d t}{t} \\
\quad+\int_{0}^{d\left(z, z^{\prime}\right) / C}\left|\int_{X}\left(\tilde{q}(x, y, t)-\tilde{q}\left(x, z^{\prime}, t\right)\right) q\left(y, z^{\prime}, t\right) d m(y)\right| \frac{d t}{t} \\
=I+I I+I I I .
\end{aligned}
$$

For $I$, we have $d(y, z) \leq C(t+d(x, z))$ so

$$
\begin{aligned}
I & \leq C \int_{d\left(z, z^{\prime}\right) / C}^{\infty} \int_{B(z, C t)} \frac{t^{\xi} d(y, z)^{\xi}}{(t+d(x, z))^{N+2 \xi}} \frac{d\left(z, z^{\prime}\right)}{t^{N+1}} d m(y) \frac{d t}{t} \\
& \leq C d\left(z, z^{\prime}\right) \int_{d\left(z, z^{\prime}\right) / C}^{\infty} \frac{t^{\xi}}{(t+d(x, z))^{N+2 \xi}} \frac{t^{N+\xi}}{t^{N+1}} \frac{d t}{t} \\
& \leq C \frac{d\left(z, z^{\prime}\right)}{d(x, z)^{N+1}}
\end{aligned}
$$

as $d\left(z, z^{\prime}\right) / C \leq d(x, z)$ and we can take $\xi>1 / 2$.

We now find a bound for $I I$, and $I I I$ is similar. As $d(y, z)<4 t$ we get $d(y, z) \leq C(t+d(x, z))$, then

$$
\begin{aligned}
I I & \leq C \int_{0}^{d\left(z, z^{\prime}\right) / C} \int_{B(z, 4 t)} \frac{t^{\xi} d(y, z)^{\xi}}{(t+d(x, z))^{N+2 \xi}} \frac{1}{t^{N}} d m(y) \frac{d t}{t} \\
& \leq C \frac{1}{d(x, z)^{N+2 \xi}} \int_{0}^{d\left(z, z^{\prime}\right) / C} t^{2 \xi} \frac{d t}{t} \\
& \leq C \frac{d\left(z, z^{\prime}\right)}{d(x, z)^{N+1}},
\end{aligned}
$$

where the last inequality also requires $\xi>1 / 2$.

- $\mathbf{Q} 1=\mathbf{Q}^{*} 1=0$ is immediate. 
- Weak boundedness: let $f, g \in C_{c}^{\gamma}(B)$ for $\gamma>0$ and $B$ a ball. For $t<C \operatorname{diam}(B), d(x, y)<c t$,

$$
\begin{aligned}
\left|\int_{X} \int_{X} \int_{X} \tilde{q}(x, y, t) q(y, z, t) f(z)(g(x)-g(y)) d m(y) d m(z) d m(x)\right| \\
\leq C\|f\|_{\infty}[g]_{\gamma} \\
\quad \times \int_{X} \int_{B} \int_{B(z, 4 t)} \frac{t^{\xi}}{(t+d(x, y))^{N+\xi}} \frac{1}{t^{N}} d(x, y)^{\gamma} d m(y) d m(z) d m(x) \\
\leq C\|f\|_{\infty}[g]_{\gamma} m(B) t^{\gamma} \\
\leq C[f]_{\gamma}[g]_{\gamma} \operatorname{diam}(B)^{N+\gamma} t^{\gamma} .
\end{aligned}
$$

And for $t \geq C \operatorname{diam}(B)$,

$$
\begin{aligned}
\left|\int_{X} \int_{X} \int_{X} \tilde{q}(x, y, t) q(y, z, t) f(z) g(x) d m(y) d m(z) d m(x)\right| \\
\leq C\|f\|_{\infty}\|g\|_{\infty} \\
\quad \times \int_{B} \int_{B} \int_{B(z, 4 t)} \frac{t^{\xi}}{(t+d(x, y))^{N+\xi}} \frac{1}{t^{N}} d m(y) d m(z) d m(x) \\
\leq C\|f\|_{\infty}\|g\|_{\infty} \frac{1}{t^{N}} m(B)^{2} \\
\leq C[f]_{\gamma}[g]_{\gamma} \operatorname{diam}(B)^{2 N+2 \gamma} t^{-N} .
\end{aligned}
$$

From these two inequalities we can conclude

$$
\begin{aligned}
|\langle\mathbf{Q} f, g\rangle| \leq & \int_{0}^{C \operatorname{diam}(B)} C[f]_{\gamma}[g]_{\gamma} \operatorname{diam}(B)^{N+\gamma} t^{\gamma} \frac{d t}{t} \\
& +\int_{C \operatorname{diam}(B)}^{\infty} C[f]_{\gamma}[g]_{\gamma} \operatorname{diam}(B)^{2 N+2 \gamma} t^{-N} \frac{d t}{t} \\
\leq & C[f]_{\gamma}[g]_{\gamma} \operatorname{diam}(B)^{N+2 \gamma} .
\end{aligned}
$$

\section{Proofs of the restriction theorems}

As mentioned in Section 2, we will start by proving Theorem 2.5.

Let $k_{\alpha}$ be the kernel of $J_{\alpha}$. In [17] it is shown that this kernel satisfies, among other properties, 
K1. $k_{\alpha} \geq 0$ and $k_{\alpha}(x, y)=k_{\alpha}(y, x)$;

K2. $k_{\alpha}(x, y) \leq C d(x, y)^{-(N-\alpha)}$;

K3. $k_{\alpha}(x, y) \leq C d(x, y)^{-(N+\alpha)}$ if $d(x, y) \geq 4$;

K4. $\left|k_{\alpha}(x, z)-k_{\alpha}(y, z)\right| \leq C d(x, y)(d(x, z) \wedge d(y, z))^{-(N+1-\alpha)}$;

and also the following integral properties:

K5. $\int_{X} k_{\alpha}(x, z) d m(z)=\int_{X} k_{\alpha}(z, y) d m(z)=1 \forall x, y$;

K6. for $q(N-\alpha)<N<q(N+\alpha)$, there exists $C>0$ such that for all $x$,

$$
\int_{X} k_{\alpha}(x, y)^{q} d m(y) \leq C
$$

K7. for $q(N-\alpha)<N<q(N-\alpha+1)$, there exists $C>0$ such that for all $x, y$,

$$
\int_{X}\left|k_{\alpha}(x, z)-k_{\alpha}(y, z)\right|^{q} d m(z) \leq C d(x, y)^{N-q(N-\alpha)} .
$$

From these, the following lemma follows. The proof is omitted.

Lemma 5.1. If $0<\alpha<1$ and $q>0$ then:

1. If $q(N-\alpha)<d<q(N+\alpha)$, there exists $C>0$ such that for $z \in X$ we have

$$
\int_{F} k_{\alpha}(s, z)^{q} d \mu(s) \leq C<\infty .
$$

2. If $q(N-\alpha)<d<q(N-\alpha+1)$ and $0<r<C \operatorname{diam}(F)$, there exists $C>0$ such that for $z \in X$ we have

$$
\int_{F} f_{B(s, r)}\left|k_{\alpha}(s, z)-k_{\alpha}(t, z)\right|^{q} d \mu(t) d \mu(s) \leq C r^{d-q(N-\alpha)} .
$$

Proof of Theorem 2.5: Define for continuous functions the operator $\mathcal{R} f=$ $\left.f\right|_{F}$. We will show that for $f$ continuous with $f=J_{\alpha} g, g \in L^{p}(X)$ the following holds:

1.

$$
\int_{F}|f(s)|^{p} d \mu(s) \leq C\|g\|_{p}^{p}
$$

2. If $0<r<C \operatorname{diam}(F)$,

$$
\int_{F} f_{B(s, r)}|f(s)-f(t)|^{p} d \mu(t) d \mu(s) \leq C r^{\beta p}\|g\|_{p}^{p} .
$$


Then

$$
\|\mathcal{R} f\|_{B_{p, \infty}^{\beta}(F, \mu)} \leq C\|f\|_{L^{\alpha, p}(X, m)},
$$

and as continuous functions are dense in $L^{\alpha, p}$ (see Section 2 or $[\mathbf{1 7}$, Corollary 4.6]), we can conclude the theorem.

We proceed now to prove both inequalities.

1. If we take $0<a<1$,

$$
\begin{aligned}
\int_{F}|f(s)|^{p} d \mu(s) & =\int_{F}\left|\int_{X} k_{\alpha}(s, y) g(y) d m(y)\right|^{p} d \mu(s) \\
\leq & \int_{F}\left(\int_{X} k_{\alpha}(s, y)^{a p}|g(y)|^{p} d m(y)\right) \\
& \times\left(\int_{X} k_{\alpha}(s, y)^{(1-a) p^{\prime}} d m(y)\right)^{p / p^{\prime}} d \mu(s) \\
\leq & C \int_{X}\left(\int_{F} k_{\alpha}(s, y)^{a p} d \mu(s)\right)|g(y)|^{p} d m(y) \\
\leq & C\|g\|_{p}^{p},
\end{aligned}
$$

where we have used the integral bounds for $k_{\alpha}$ found at the beginning of the section (property K6) and Lemma 5.1. This can be done as long as $0<a<1$ satisfies $(1-a) p^{\prime}(N-\alpha)<N<(1-a) p^{\prime}(N+\alpha)$ and $a p(N-\alpha)<d<a p(N+\alpha)$, or equivalently

$$
\frac{d}{p(N+\alpha)}<a<\frac{d}{p(N-\alpha)}
$$

and

$$
\frac{N-\alpha p}{p(N-\alpha)}<a<\frac{N+\alpha p}{p(N+\alpha)},
$$

and such an $a$ can always be found, as $d<N+\alpha p$ and $N-\alpha p<d$.

2. Once more take $0<a<1$. Given $s, t \in F$,

$$
\begin{aligned}
|f(s)-f(t)|^{p} \leq & \left(\int_{X}\left|k_{\alpha}(x, s)-k_{\alpha}(x, t)\right||g(x)| d m(x)\right)^{p} \\
\leq & \left(\int_{X}\left|k_{\alpha}(x, s)-k_{\alpha}(x, t)\right|^{a p}|g(x)|^{p} d m(x)\right) \\
& \times\left(\int_{X}\left|k_{\alpha}(x, s)-k_{\alpha}(x, t)\right|^{(1-a) p^{\prime}} d m(x)\right)^{p / p^{\prime}} .
\end{aligned}
$$


Then if $d(s, t)<r$, from property $\mathbf{K} \mathbf{7}$ we have

$$
\int_{X}\left|k_{\alpha}(x, s)-k_{\alpha}(x, t)\right|^{(1-a) p^{\prime}} d m(x) \leq C r^{N-(1-a) p^{\prime}(N-\alpha)}
$$

if $0<a<1$ satisfies $(1-a) p^{\prime}(N-\alpha)<N<(1-a) p^{\prime}(N-\alpha+1)$. Now,

$$
\begin{aligned}
& \int_{F} f_{B(s, r)}|f(s)-f(t)|^{p} d \mu(t) d \mu(s) \leq C r^{N \frac{p}{p^{\prime}}-(1-a) p(N-\alpha)} \\
& \quad \times \int_{X}\left(\int_{F} f_{B(s, r)}\left|k_{\alpha}(x, s)-k_{\alpha}(x, t)\right|^{a p} d \mu(t) d \mu(s)\right)|g(x)|^{p} d m(x)
\end{aligned}
$$

and therefore by Lemma 5.1

$$
\int_{F} f_{B(s, r)}\left|k_{\alpha}(x, s)-k_{\alpha}(x, t)\right|^{a p} d \mu(t) d \mu(s) \leq C r^{d-a p(N-\alpha)}
$$

whenever $a$ satisfies $a p(N-\alpha)<d<a p(N-\alpha+1)$ and we conclude

$$
\begin{aligned}
\int_{F} f_{B(s, r)}|f(s)-f(t)|^{p} d \mu(t) d \mu(s) & \leq C r^{N \frac{p}{p^{\prime}}-(1-a) p(N-\alpha)} r^{d-a p(N-\alpha)}\|g\|_{p}^{p} \\
& =C r^{p \beta}\|g\|_{p}^{p} .
\end{aligned}
$$

The existence of this $a$ is equivalent to

$$
\frac{d}{p(N+1-\alpha)}<a<\frac{d}{p(N-\alpha)}
$$

and

$$
\frac{N-\alpha p}{p(N-\alpha)}<a<\frac{N+p-\alpha p}{p(N+1-\alpha)}
$$

These intervals are well defined, as $p(N+1-\alpha)>p(N-\alpha)$ and

$$
\begin{aligned}
(N-\alpha p)(N+1-\alpha) & =N^{2}-N \alpha p+N-\alpha p-N \alpha+\alpha^{2} p \\
& <N^{2}-N \alpha+N p-\alpha p-N \alpha p+\alpha^{2} p \\
& =(N-\alpha)(N+p-\alpha p)
\end{aligned}
$$

and they overlap, as $N-\alpha p<d$ and $d<N+p-\alpha p$.

We are now ready to prove the restriction theorem for Besov functions. 
Proof of Theorem 2.6: Using Theorem 2.5 for $0<\alpha_{1}, \alpha_{2}<1$ and their corresponding $\beta_{1}, \beta_{2}$, and the fact that $\left(B_{p, \infty}^{\beta_{1}}, B_{p, \infty}^{\beta_{2}}\right)_{\theta, q} \hookrightarrow B_{p, q}^{\beta}$ (see Lemma 4.1), we obtain that the restriction operator $\mathcal{R}$ is well defined and continuous

$$
\mathcal{R}:\left(L^{\alpha_{1}, p}, L^{\alpha_{2}, p}\right)_{\theta, q} \rightarrow B_{p, q}^{\beta}(F, \mu),
$$

where $\beta=(1-\theta) \alpha_{1}+\theta \alpha_{2}-\frac{N-d}{p}$.

Now, as for the case when $0<\alpha_{1}, \alpha_{2}<\alpha_{0}$ we have a characterization for $\left(L^{\alpha_{1}, p}, L^{\alpha_{2}, p}\right)_{\theta, q}$, using the interpolation result (Theorem 2.4) we can conclude the restriction theorem.

\section{Acknowledgements}

The author is infinitely indebted to his advisors Eleonor 'Pola' Harboure and Hugo Aimar for their guidance and support throughout the development of his doctoral thesis. Specially to Pola for her help and invaluable advice during the production and improvement of the papers resulting from the thesis.

He would also like to thank the referees for their many suggestions to improve this article.

\section{References}

[1] H. Aimar, "Distance and Measure in Analysis and Partial Differential Equations", in preparation.

[2] H. Aimar, M. Carena, R. Durán, and M. Toschi, Powers of distances to lower dimensional sets as Muckenhoupt weights, Acta Math. Hungar. 143(1) (2014), 119-137. DOI: 10.1007/s10474-0140389-1.

[3] C. Bennett And R. Sharpley, "Interpolation of Operators", Pure and Applied Mathematics 129, Academic Press, Inc., Boston, MA, 1988.

[4] J. Bergh AND J. LÖFström, "Interpolation Spaces. An Introduction", Grundlehren der Mathematischen Wissenschaften 223, Springer-Verlag, Berlin-New York, 1976. DOI : 10.1007/978-3-64266451-9.

[5] R. R. CoIfman And G. WeIss, "Analyse harmonique non-commutative sur certains espaces homogènes. Étude de certaines intégrales singulières", Lecture Notes in Mathematics 242, Springer-Verlag, Berlin-New York, 1971. DOI: 10.1007/BFb0058946. 
[6] A. E. GatTo, On fractional calculus associated to doubling and non-doubling measures, in: "Harmonic Analysis: CalderónZygmund and Beyond", Contemp. Math. 411, Amer. Math. Soc., Providence, RI, 2006, pp. 15-37. DOI: 10.1090/conm/411/07745.

[7] A. E. Gatto, C. Segovia, And S. VÁgi, On fractional differentiation and integration on spaces of homogeneous type, Rev. Mat. Iberoamericana 12(1) (1996), 111-145. DOI: 10.4171/RMI/196.

[8] A. Gogatishvili, P. Koskela, and N. Shanmugalingam, Interpolation properties of Besov spaces defined on metric spaces, Math. Nachr. 283(2) (2010), 215-231. DOI: 10.1002/mana. 2008 10242.

[9] A. Gogatishvili, P. Koskela, and Y. Zhou, Characterizations of Besov and Triebel-Lizorkin spaces on metric measure spaces, Forum Math. 25(4) (2013), 787-819.

[10] Y. S. HAN AND E. T. SAWYER, "Littlewood-Paley Theory on Spaces of Homogeneous Type and the Classical Function Spaces", Mem. Amer. Math. Soc. 530, Amer. Math. Soc., Providence, RI, 1994. DOI : 10.1090/memo/0530.

[11] T. Heikkinen, L. Ihnatsyeva, And H. Tuominen, Measure density and extension of Besov and Triebel-Lizorkin functions, $J$. Fourier Anal. Appl. 22(2) (2016), 334-382. DOI: 10.1007/s00041015-9419-9.

[12] J. Heinonen, "Lectures on Analysis on Metric Spaces", Universitext, Springer-Verlag, New York, 2001. DOI : 10.1007/978-1-46130131-8.

[13] A. Jonsson And H. Wallin, Function spaces on subsets of $\mathbf{R}^{n}$, Math. Rep. 2(1) (1984), 221 pp.

[14] R. A. Macías And C. Segovia, Lipschitz functions on spaces of homogeneous type, Adv. in Math. 33(3) (1979), 257-270. DOI: 10.1016/0001-8708 (79) 90012-4.

[15] R. A. Macías and C. Segovia, A decomposition into atoms of distributions on spaces of homogeneous type, Adv. in Math. 33(3) (1979), 271-309. DOI : 10.1016/0001-8708(79)90013-6.

[16] J. MALÝ, Coarea integration in metric spaces, in: "NAFSA 7Nonlinear Analysis, Function Spaces and Applications", Vol. 7, Czech. Acad. Sci., Prague, 2003, pp. 148-192.

[17] M. A. Marcos, Bessel potentials in Ahlfors regular metric spaces, Potential Anal. 45(2) (2016), 201-227. DOI: 10.1007/s11118-0169543-4. 
[18] D. Müller AND D. YANG, A difference characterization of Besov and Triebel-Lizorkin spaces on RD-spaces, Forum Math. 21(2) (2009), 259-298. DOI: 10.1515/FORUM.2009.013.

[19] J. Peetre, "New Thoughts on Besov Spaces", Duke University Mathematics Series 1, Mathematics Department, Duke University, Durham, N.C., 1976.

[20] E. M. Stein, "Singular Integrals and Differentiability Properties of Functions", Princeton Mathematical Series 30, Princeton University Press, Princeton, N.J., 1970.

Instituto de Matemática Aplicada del Litoral

UNL, CONICET, FIQ

Predio Dr. Alberto Cassano del CCT-CONICET-Santa Fe

Colectora de la Ruta Nacional No 168, Santa Fe

Argentina

E-mail address: mmarcos@santafe-conicet.gov.ar

Primera versió rebuda el 27 de juliol de 2016, darrera versió rebuda el 6 de març de 2017. 